\title{
Exercício físico e esportes no combate à obesidade infantil
}

\author{
Physical exercise sports to fight childhood obesity \\ Ejercicio físico para combatir la obesidad infantil
}

Recebido: 07/07/2021 | Revisado: 10/07/2021 | Aceito: 12/07/2021 | Publicado: 22/07/2021

\author{
Sebastião Lobo da Silva \\ ORCID: https://orcid.org/0000-0003-2639-0898 \\ Universidade Católica de Brasília, Brasil \\ E-mail: slobo2011@gmail.com \\ Tiago Teixeira de Queiroz \\ ORCID: https://orcid.org/0000-0003-1541-9085 \\ Faculdade de Piracanjuba, Brasil \\ E-mail: queiroztiago658@gmail.com \\ Raquel Borges de Souza \\ ORCID: https://orcid.org/0000-0003-1675-6649 \\ Faculdade de Piracanjuba, Brasil \\ E-mail: raquelsab1980@gmail.com \\ Givanildo de Oliveira Santos \\ ORCID: https://orcid.org/0000-0001-5279-1535 \\ Faculdade de Mauá Brasília, Brasil \\ E-mail: givanildo-o@hotmail.com \\ Carmen Silvia Grubert Campbell \\ ORCID: https://orcid.org/0000-0001-5946-7180 \\ Universidade Católica de Brasília, Brasil \\ E-mail: campbellcsg@gmail.com
}

\begin{abstract}
Resumo
Dentre os fatores que contribuem para a obesidade infantil estão a alimentação não saudável e a falta de exercício físico. O objetivo deste estudo foi avaliar o desenvolvimento da obesidade infantil e os estudos a respeito do exercício físico e esportes utilizados no combate à obesidade infantil. A presente pesquisa trata de uma revisão bibliográfica descritiva. Para o levantamento bibliográfico foram utilizados artigos científicos disponíveis on-line entre os anos 2003 e 2019. A busca dos artigos foi realizada na base de dados do Google acadêmico e PubMed no período de agosto a janeiro de 2021. A prática de exercício físico e esportes promoveu maior gasto energético, benefícios metabólicos, controle da frequência cardíaca, melhora da função cardiorrespiratória, das habilidades locomotoras, lateralidade, resistência e redução do índice de massa corpórea em crianças.
\end{abstract}

Palavras-chave: Educação física; Dieta; Alimentação; Comportamento sedentários; Crianças.

\begin{abstract}
Among the factors that contribute to childhood obesity are unhealthy eating and lack of physical exercise. The aim of this study was to assess the development of childhood obesity and studies on physical exercise and sports used to combat childhood obesity. This research is a descriptive bibliographic review. Scientific articles available online between 2003 and 2019 were used for the bibliographic survey. The search for articles was performed in the academic Google database and PubMed from August to January 2021. The practice of physical exercise and sports promoted greater energy expenditure, metabolic benefits, heart rate control, improvement in cardiorespiratory function, locomotor skills, laterality, endurance and reduced body mass index in children.
\end{abstract}

Keywords: Physical education; Diet; Food; Sedentary behavior; Kids.

\section{Resumen}

Entre los factores que contribuyen a la obesidad infantil se encuentran la alimentación poco saludable y la falta de ejercicio físico. El objetivo de este estudio fue evaluar el desarrollo de la obesidad infantil y estudios sobre el ejercicio físico y el deporte utilizados para combatir la obesidad infantil. Esta investigación es una revisión bibliográfica descriptiva. Para la encuesta bibliográfica se utilizaron artículos científicos disponibles en línea entre 2003 y 2019. La búsqueda de artículos se realizó en la base de datos académica de Google y PubMed de agosto a enero de 2021. La práctica de ejercicio físico y deporte promovió un mayor gasto energético, beneficios metabólicos, cardíacos control de frecuencia, mejora de la función cardiorrespiratoria, habilidades locomotoras, lateralidad, resistencia e índice de masa corporal reducido en niños.

Palabras clave: Educación física; Dieta; Alimentación; Comportamiento sedentario; Niños. 


\section{Introdução}

$\mathrm{O}$ exercício físico e a atividade física podem auxiliar no combate à obesidade infantil, porém muitas crianças e adolescentes não aderem aos mesmos se tornando sedentários. Aliado a isso, a alimentação rica em carboidratos, açúcares, gorduras alimentos contribuem para o aumento de peso nesta faixa etária. Por estes fatos a saúde pública se mostra preocupada com a saúde infantil e com as consequências que possam surgir com o aumento de peso (Oliveira \& Fisberg, 2003).

Em virtude dos riscos proeminentes de obesidade em todas as faixas etárias para a saúde, busca-se uma estratégia de saúde pública com profissionais capacitados para se determinar uma conduta para a prevenção e o diagnóstico da obesidade. A conscientização de crianças e seus responsáveis sobre o risco que hábitos nocivos exercem sobre a saúde, e que estes podem ser modificados a partir da introdução de uma alimentação mais saudável e da prática de atividades que sejam prazerosas e que aumentem o gasto energético (Santos et al., 2021).

A Educação Física dentro do âmbito escolar procura garantir que as crianças busquem se mover, brincar, se divertir, de maneira que as crianças possam sentir prazer, alegria e bem-estar, por meio do desenvolvimento de habilidades e capacidades físicas. Através da Educação Física na escola, a importância de praticar atividades físicas regularmente pode ser transmitida para crianças e adolescentes contribuindo para melhorar seus hábitos e a redução da incidência de obesidade nesta faixa etária (Benedito et al., 2015).

Objetivou-se neste estudo verificar as atividades praticadas de forma prazerosas por crianças como estratégias para a redução da obesidade infantil.

\section{Metodologia}

De acordo com Estrela (2018), a presente pesquisa trata-se de uma revisão bibliográfica de natureza qualitativa, para o levantamento bibliográfico foram utilizados artigos científicos disponíveis online em formato de pdf, entre os anos (20032019). A busca dos artigos foi realizada na base de dados do Google acadêmico no período de agosto/2020 a janeiro/2021. Para a busca literária foram utilizados os seguintes descritores: Educação física, obesidade infantil, a importância da educação física, benefícios promovidos pela prática de exercícios físicos no combate à obesidade.

\section{Revisão}

\subsection{Obesidade}

A obesidade é uma doença não transmissível que afeta grande parte da população mundial e resulta no acúmulo de gordura em excesso e diversos prejuízos à saúde. Uma pessoa portadora de obesidade está mais propensa a apresentar sintomas de doenças como hipertensão arterial, diabetes, distúrbios na locomoção e no aparelho motor, dificuldades respiratórias e cardiovasculares (Pinheiro et al., 2004).

A obesidade é uma doença multifatorial envolvendo condições ambientais e genéticas de determinada população, como má alimentação, disfunções endócrinas, sedentarismo, sendo considerada como preocupação em toda a população mundial (Pinheiro et al., 2004).

Para Oliveira e Fisberg (2003), o ganho de peso de maneira excessiva em curto período resulta em alteração na estatura da idade óssea, uma puberdade mais precoce, podendo ocasionar uma estatura diminuída em relação à altura pelo fechamento precoce das cartilagens relacionadas ao crescimento. Para estes autores, o excesso de peso na infância pode ser detectado pelo método de avaliação do Índice de Massa Corpórea (IMC) obtido a partir do peso / altura ${ }^{2}$, bem como pela medida da dobra cutânea (Oliveira \& Fisberg, 2003).

O estilo de vida, o sedentarismo e o mau hábito alimentar contribui para o desenvolvimento da obesidade. A vida 
contemporânea com muitos afazeres e tempo escasso favorece uma alimentação de baixa qualidade com alimentos ricos em gordura, açúcares simples e industrializados (Wanderley \& Ferreira, 2010).

Medidas de prevenção e controle do número de casos da obesidade por meio de intervenções envolvendo dieta e exercício físico têm sido utilizadas como estratégias de saúde para redução dos casos de obesidade pelo mundo (Triches \& Giugliani, 2005). Apesar da obesidade ser considerada um dos principais fatores de desordem nutricional, a eficácia do tratamento necessita também do acompanhamento psicológico para uma melhor compreensão do aspecto emocional que geralmente aflige os portadores de obesidade (Wanderley \& Ferreira, 2010), além do trabalho multidisciplinar com outros profissionais da área da saúde como professor de educação física, nutricionista e endocrinologista.

Neste contexto, torna-se interessante a definição de estratégias da secretaria da saúde para o controle da obesidade, principalmente em crianças (Mello, et al., 2004; Triches \& Giugliani, 2005).

A educação, renda, ocupação podem contribuir para o ganho de peso e redução do gasto energético, da taxa metabólica de repouso e padrões comportamentais de forma diferente de acordo com a classe social (Mello, et al., 2004).

Estratégias de conscientização sobre a importância de se ter uma vida saudável e a necessidade de se praticar uma atividade física estimulando uma maior movimentação dessa criança visando o aumento do gasto energético, de uma alimentação balanceada e saudável, incluindo a ingestão de frutas, saladas, verduras, carnes magras, e redução do consumo de alimentos ricos em frituras, gorduras saturadas e totais. Esse trabalho de conscientização não deve ser apenas das crianças, mas também dos pais ou responsáveis, pois os hábitos das crianças geralmente são os mesmos das pessoas de sua convivência (Mello, et al., 2004).

\subsection{Exercícios físicos e esportes no combate da obesidade infantil}

A Educação Física no âmbito escolar é importante para o combate da obesidade infantil, pois são nessas aulas que as crianças se movimentam por meio de atividades físicas prazerosas, de lazer e esportiva contribuindo de forma eficaz para a redução do sedentarismo, desenvolvimento do interesse e gosto pelo esporte, estímulo ao estilo de vida saudável e consciente para prevenção da obesidade infantil e de doenças a ela associadas (Benedito, et al., 2015).

Para Benedito, et al. (2015), as aulas de Educação Física se tornaram um dos poucos lugares em que as crianças praticam algum tipo de atividade física, além de ser um local de oportunidade para desenvolver temas relacionados à saúde no sentido de melhorar a qualidade de vida das crianças.

As aulas de Educação Física podem favorecer um maior engajamento na prática de atividades físicas em crianças que, de acordo com Bravin, et al., (2015), quando realizada regularmente exerce papel primordial na prevenção da obesidade infantil.

Poeta, et al., (2012) verificaram que o grupo intervenção que participou das atividades lúdicas reduziu o índice de massa corpórea, as dobras cutâneas e circunferência abdominal quando comparado ao grupo controle que apresentou ainda um aumento da massa corporal e circunferência do abdômen. Para Santos (2019), a atividade lúdica, através de jogos e brincadeiras, contribui para o desenvolvimento de habilidades motoras e o aprendizado escolar, e para Gumieri (2016), este tipo de atividade auxilia nos aspectos cognitivo, físico, afetivo e social da criança.

Sabia, et al., (2004), verificaram redução do índice de massa corpórea e melhora cardiorrespiratória em crianças após prática de atividade física associada com alimentação saudável. Para Paes, et al., (2015), a prática de atividade física promoveu benefícios na restauração do perfil lipídico de crianças, melhora da composição corporal, do sistema cardiovascular e ativação metabólica.

Landucci (2004), usou um programa com atividades aeróbias com duração de 45 a 50 minutos, 3 vezes por semana, durante três meses em crianças, resultando em redução da gordura corporal, melhora do desempenho cardiorrespiratório e da 
aptidão física. Silva (2004), observou melhora no condicionamento cardiorrespiratório e da composição corporal após um período de três meses de ciclismo indoor com frequência de três vezes por semana, com duração de 50 minutos, mais alongamento e caminhada em um programa de integração na redução de peso em crianças e jovens.

Palma, et al., (2012), observaram desempenho motor superior em praticantes de atividades físicas quando comparados aos não praticantes. Mello, et al., (2004), verificaram redução do sedentarismo e no índice da obesidade infantil após participação em um programa de seis meses de avaliação em ambas estratégias, atendimento ambulatorial (individual) e programa de educação (em grupo).

Leite, et al., (2010), constataram uma redução do índice de massa corpórea e melhora da função cardiorrespiratória após participação em programa de natação realizado três vezes por semana totalizando 36 sessões em 12 semanas. No estudo de Santos (2015), a atividade aquática para membros superiores e inferiores realizada em três sessões semanais, reduziu o índice de massa corpórea, promoveu melhoras em variáveis bioquímicas e hemodinâmicas (colesterol e insulina), além da dimensão psicossocial. De acordo com De Melo, et al., (2020), os exercícios de natação contribuem para um melhor desenvolvimento da criança e adolescentes tanto no aspecto motor, psicológico e social e, portanto, para o controle e redução da obesidade infantil.

Casagrande (2014), sugere que o jump kids pode ser considerado como uma atividade física para auxiliar no combate da obesidade infantil. Benefícios na redução do índice de massa corpórea, melhora da capacidade cardiorrespiratória, lateralidade e equilíbrio bem como maior amplitude de movimento foram observados após intervenção com três aulas semanais com duração em média de 45 minutos, por um período de 12 semanas. Para De Lima, et al., (2017), no seu estudo usou uma amostra com 47 jovens e adolescentes três vezes por semana com duração de 12 semanas, variando o tempo entre 24 e 32 minutos; houve diminuição do índice de massa corpórea e diminuindo a taxa de obesidade.

Faude, et al., (2010), relataram melhora das habilidades motoras, da resposta cardiovascular e da frequência cardíaca submáxima após prática de futebol três vezes na semana. De acordo com Pedretti, et al., (2016), a prática do futebol reduziu fatores de riscos metabólicos, melhorou a composição corporal contribuindo, portanto para a prevenção da obesidade na infância. Para Oliveira, et al., (2021), o exercício físico bem planejado alinhado aos objetivos das crianças e adolescentes poderá resultar em ganho de massa muscular, redução no tecido adiposo e do peso corporal total. Owen, et al., (2012), relataram que o futebol realizado de 3 a 4 vezes por semana, em intensidade moderada à vigorosa, promoveu melhora da funcionalidade redução do índice de massa corpórea, e aumento do gasto calórico.

Além desses benefícios da prática do futebol na infância, Fernandes, et al., (2015), consideraram que o futebol praticado duas vezes na semana com duração de 60 minutos contribuiu para melhora da resistência e da velocidade das crianças.

\section{Considerações Finais}

O desenvolvimento de atividades prazerosas, atividades lúdicas, caminhada, corrida, natação, futebol, ciclismo indoor e jump demostraram trazer benefícios à saúde das crianças em curto período de tempo, podendo contribuir como estratégias para a redução do avanço de casos de obesidade na infância.

A prática do exercício físico promove o interesse das crianças na prática de alguma atividade física e, com isso, estimula o gasto energético, a melhora do metabolismo, o controla das funções cardiovascular e respiratória, além de contribuir para o desenvolvimento de habilidades motoras, equilíbrio, lateralidade, resistência, a amplitude de movimento, força, redução do índice de massa corpórea e do sedentarismo, e consequentemente da saúde infantil de maneira geral.

Após o levantamento bibliográficos realizados de natureza qualitativa, sugerimos para futuras pesquisas uma revisão sistematizada de natureza quantitativa, para que possa mensurar estatisticamente os resultados de estudos aplicados a 
voluntários em pesquisas com intervenção.

\section{Referências}

Benedito, L. D. S., Pagani, M. M., Gomes, I. D. S., \& Avila, R. N. P. (2015). Educação física escolar: no combate à obesidade infantil. Trabalho de Conclusão de Curso na FAEMA. http://repositorio.faema.edu.br:8000/jspui/handle/123456789/2196

Bravin, M. B., Rosa, A. R., Parreira, M. B., \& Prado, A. F. (2015). A influência do exercício físico na obesidade infantil. Revista Ciência e Estudos Acadêmicos de Medicina, 1 (04).

Casagrande, A. R. (2014). Avaliação da composição corporal em crianças com sobrepeso durante uma metodologia com aulas de jump kids. Trabalhos de Conclusão de Curso apresentado ao Curso de Licenciatura em Educação Física, do Instituto Federal de Educação Ciência e Tecnologia do Sul de Minas Gerais (IFSul de Minas) - Câmpus Muzambinho).

De Lima Magalhães, R., dos Santos, N. S., da Costa, R. F., Bones, V., Fernando, L., \& Kruel, M. (2017). Efeitos de dois tipos de treinamento físico de baixo impacto sobre o tempo de tela em adolescentes com excesso de peso. J Hum Growth Dev, 27 (3), 294-299.

De Lima Neves Barros, M. (2012). Exergames: o papel multidisciplinar do design no desenvolvimento de jogos de exercício físico-funcional para o auxílio no combate da obesidade infantil (Master's thesis, Universidade Federal de Pernambuco).

De Melo, J. M. P., Souza, J. R., Lima, R. K. V., da Silva, S. L., \& de Oliveira Santos, G. (2020). Benefícios da natação para crianças e adolescentes. Brazilian Journal of Development, 6(8), 62511-62519.

Estrela, C. (2018). Metodologia Científica: Ciência, Ensino, Pesquisa. Editora Artes Médicas.

Faude, O., Kerper, O., Multhaupt, M., Winter, C., Beziel, K., Junge, A., \& Meyer, T. (2010). Football to tackle overweight in children. Scandinavian journal of medicine \& science in sports, 20, 103-110.

Fernandes, L., Oliveira, J., Soares-Miranda, L., Rebelo, A., \& Brito, J. (2015). Regular football practice improves autonomic cardiac function in male children. Asian journal of sports medicine, 6 (3).

Fernandez, A. C., Mello, M. T. D., Tufik, S., Castro, P. M. D., \& Fisberg, M. (2004). Influência do treinamento aeróbio e anaeróbio na massa de gordura corporal de adolescentes obesos. Revista Brasileira de Medicina do Esporte, 10(3), 152-158.

GUMIERI, F. A. (2016). A importância do lúdico para o desenvolvimento da criança: o brincar como ferramenta de aprendizagem na Educação Infantil. Cadernos de Educação: Ensino e Sociedade, Bebedouro - SP, 3 (1): 66 - 80.

Landucci, M. B. (2004). Os benefícios da caminhada no tratamento da obesidade juvenil. Trabalho de Conclusão de Curso na UFPR. http: https://www.acervodigital.ufpr.br/handle/1884/47636

Leite, N., Lazarotto, L., Cavazza, J. F., Lopes, M. D. F. A., Bento, P. C. B., Torres, R., ... \& Milano, G. E. (2010). Effects of aquatic exercise and nutritional guidance on the body composition of obese children and adolescents. Revista Brasileira de Cineantropometria \& Desempenho Humano, 12(4), $232-238$.

Mello, E. D. D., Luft, V. C., \& Meyer, F. (2004). Atendimento ambulatorial individualizado versus programa de educação em grupo: qual oferece mais mudança de hábitos alimentares e de atividade física em crianças obesas? Jornal de Pediatria, 80(6), 468-474.

Oliveira, C. L. D., \& Fisberg, M. (2003). Obesidade na infância e adolescência: uma verdadeira epidemia. Arquivos Brasileiros de Endocrinologia \& Metabologia, 47(2), 107-108.

Owen, A. L., Wong, D. P., Paul, D., \& Dellal, A. (2012). Effects of a periodized small-sided game training intervention on physical performance in elite professional soccer. The Journal of Strength \& Conditioning Research, 26(10), 2748-2754.

Paes, S. T., Marins, J. C. B., \& Andreazzi, A. E. (2015). Efeitos metabólicos do exercício físico na obesidade infantil: uma visão atual. Revista Paulista de Pediatria, 33(1), 122-129.

Palma, M. S., Camargo, V. A. D., \& Pontes, M. F. P. (2012). Efeitos da atividade física sistemática sobre o desempenho motor de crianças pré-escolares. Revista da Educação Física/UEM, 23(3), 421-429.

Pedretti, A., Pedretti, A., Vasconcellos, F., \& Seabra, A. (2016). O futebol recreativo como uma nova abordagem terapêutica para a obesidade em crianças e adolescentes: uma revisão. Revista Brasileira de Atividade Física \& Saúde, 21(2), 123-132.

Pinheiro, A. R. D. O., Freitas, S. F. T. D., \& Corso, A. C. T. (2004). Uma abordagem epidemiológica da obesidade. Revista de Nutrição, 17(4), 523-533.

Poeta, L. S., da Silva Duarte, M. D. F., Giuliano, I. D. C. B., \& de Farias Junior, J. C. (2012). Interdisciplinary intervention on body composition and physical fitness tests in obese children. Brazilian Journal of Kinanthropometry and Human Performance, 14(2), 134-143.

Sabia, R. V., Santos, J. E. D., \& Ribeiro, R. P. P. (2004). Efeito da atividade física associada à orientação alimentar em adolescentes obesos: comparação entre o exercício aeróbio e anaeróbio. Revista Brasileira de Medicina do Esporte, 10 (5), 349-355.

Santos, A. A., \& Pereira, O. J. (2019). A importância dos jogos e brincadeiras lúdicas na Educação Infantil. Revista Eletrônica Pesquiseduca, 11(25), 480-493.

Santos de Oliveira G., Bagestão, V. S., \& da Silva, S. L. (2021). Efeitos dos exercícios físicos em crianças e adolescentes. Brazilian Journal of Development, $7(1), 8903-8915$. 
Research, Society and Development, v. 10, n. 9, e13710917980, 2021

(CC BY 4.0) | ISSN 2525-3409 | DOI: http://dx.doi.org/10.33448/rsd-v10i9.17980

Santos, N. S. D. (2015). Efeitos do treinamento aquático e terrestre sobre os fatores de risco cardiometabólico, qualidade de vida e saúde mental em adolescentes com excesso de peso: ensaio clínico randomizado. Dissertação de mestrado da UFRGS. https://www.lume.ufrgs.br/handle/10183/133612

Silva, T. D. O. (2004). Ciclismo indoor para crianças e adolescentes obesos. Trabalho de Conclusão de Curso na UFPR. https://www.acervodigital.ufpr.br/handle/1884/47662.

Triches, R. M., \& Giugliani, E. R. J. (2005). Obesidade, práticas alimentares e conhecimentos de nutrição em escolares. Revista de Saúde Pública, 39, 541547.

Wanderley, E. N., \& Ferreira, V. A. (2010). Obesity: a plural perspective. Ciencia \& saude coletiva, 15(1), 185-194. 